\title{
EFECTOS DE UN PROGRAMA PARA DESARROLLAR LA INTELIGENCIA EMOCIONAL EN ALUMNOS DEL SEXTO GRADO DE EDUCACIÓN PRIMARIA
}

\author{
Amparo Sotil B. ${ }^{1}$, luis Escurra M., Rosa Huerta, Marcelina Rosas C., Emma Campos P., Ana Llaños \\ Universidad Nacional Mayor de San Marcos, Lima, Perú \\ (RECIBIDO EL 31/10/2008, ACEPTADO EL 2/12/2008)
}

\begin{abstract}
RESUMEN
Objetivos: Efectos de un programa para desarrollar la Inteligencia Emocional en alumnos del sexto grado de educación primaria. Diseño: Estudio experimental con diseño cuasiexperimental, realizado en alumnos del sexto grado de educación primaria de la ciudad de Lima en el 2005. Material y métodos: Muestreo aplicado de tipo intencional; la muestra para el estudio estuvo conformada con 80 estudiantes; 40 de colegios estatales y 40 de colegios no estatales; en cada caso 20 conformaron el grupo experimental y 20 el grupo de control. Se utilizó el inventario de Inteligencia Emocional de Barón ICE:NA. Se aplicó un programa para el incremento de la inteligencia emocional de los alumnos, desarrollando las siguientes actividades: A) implementación de un programa dirigido a los alumnos en el cual se trabajó las habilidades y competencias que constituyen la inteligencia emocional. B) Se organizó un ciclo de conferencias para los docentes sobre la Inteligencia Emocional y su importancia en el trabajo con los alumnos. C) Teniendo en cuenta la importancia de la participación activa de los padres de familia, se organizó un ciclo de conferencias sobre Inteligencia emocional y la importancia en el trabajo con sus hijos. El análisis estadístico se desarrolló en dos etapas, el ANÁLISIS DESCRIPTIVo de las variables estudiadas y el ANÁLISIS INFERENCIAL BIVARIADO para contrastar las hipótesis planteadas. Resultados: La media de la edad de los alumnos evaluados fue de 11,1 \pm 1,40 años, el 59,9\% fueron mujeres y el $40,5 \%$ varones. Los contrastes de hipótesis indicaron que vistieron diferencias a favor del grupo experimental en las áreas y el total de la inteligencia emocional, mientras que no se encontraron diferencias estadísticas significativas por el tipo de colegio ni por sexo. Conclusiones: Los hallazgos indican que el programa fue eficiente para incrementar la inteligencia emocional en los alumnos que participaron en el grupo experimental. No se apreciaron estadísticas significativas en las comparaciones por el tipo de gestión del colegio de procedencia y el sexo de los alumnos.
\end{abstract}

Palabras clave: Programa inteligencia emocional, educación primaria.

1 Docente Principal de la Facultad de Psicología de la UNMSM. E-mail: asotil@unmsm.edu.pe. 


\begin{abstract}
Objective: Effects of a program to develop the Emotional Intelligence in pupils of the sixth degree of primary education. Design: Experimental study with design cuasi experimental, realized in pupils of the sixth degree of primary education of the city of Lima in 2005. Material and methods: Applied Sampling was of the intentional one; the sample for the study was shaped by 80 students; 40 of state colleges and 40 of not state colleges, in every case 20 shaped the experimental group and 20 the group of control. There was in use the inventory of Baron ICE:NA's Emotional Intelligence. A program was applied for increase of the emotional intelligence of the pupils in which the following activities developed: A) implementation of a program directed the pupils at which one was employed the skills and competitions that constitute the emotional intelligence. B) One organized a cycle of conferences for the teachers on the Emotional Intelligence and his (her, your) importance in the work with the pupils. C) Having in it (he, she) counts (tells) the importance of the active participation of the family parents, one organized a cycle of conferences on emotional Intelligence and the importance in the work with his (her, your) children. The statistical analysis developed in two stages, the DESCRIPTIVE ANALYSIS of the studied variables and the ANALYSIS INFERENCIAL BIVARIADO to confirm the raised hypotheses. Results: The average of the age of the evaluated pupils was of $11.1 \pm 1.40$ years, $59,9 \%$ was women and 40,5\% was males. The contrasts of hypothesis indicated that they dressed differences in favour of the experimental group in the areas and the whole of the emotional intelligence, whereas they found statistical significant differences neither for the type of college nor for sex. Conclusions: The finds indicate that the program was efficient to increase the I. E. in pupils who took part in the experimental group. They did not appreciate significant statistics in the comparisons for the type of management of the college of origin and the sex of the pupils
\end{abstract}

Keywords: Program Emotional intelligence, Primary education.

\title{
INTRODUCCIÓN
}

El estudio tuvo por finalidad implementar un programa para desarrollar las capacidades que son de suma importancia en la Inteligencia Emocional en alumnos de sexto grado de primaria.

Se trató de contribuir para que la práctica cotidiana en el aula brinde una experiencia satisfactoria que genere motivación y disciplina, que contemple que la educación trascienda a otorgar felicidad y dar sentido a la vida, para amar y ser amado, para vivir con esperanza y descubrir quién es. Esto ha de ser lo "más importante", lo esencial, el fundamento de nuestra acción educativa.

En la actualidad se han llevado a cabo pocos estudios que, en forma sistemática, trabajen con el docente de aula, tutores y padres de familia, utilizando el programa como la estrategia de trabajo que permita desarrollar y fortalecer la inteligencia emocional de los educandos, lo cual no se considera en el currículo de estudios.

Salovey y Mayer (1990) proponen que la Inteligencia Emocional es la habilidad de manejar los sentimientos y emociones, determinar entre ellos y utilizar estos conocimientos para dirigir los propios pensamientos y acciones. Goleman (1995), con su obra inteligencia emocional, invita a reflexionar sobre las necesidades emocionales y difunde el tema en diversos países. Para él la Inteligencia Emocional es la habilidad para motivarse y persistir 
frente a las frustraciones, controlar los impulsos y postergar las gratificaciones, regular los estados de humor, evitar que las desgracias obstaculicen la habilidad de pensar, desarrollar empatía y optimismo.

Baron (1997), siguiendo el enfoque no cognitivo, relaciona los aspectos no intelectivos propuestos por Wechsler (1996) y, tomando como base la propuesta de Salovey y Mayer, nos brinda una definición operacional. Hoy día su propuesta se considera la más amplia y adecuada a la literatura de las habilidades emocionales.

Gardner (1995) desarrolla una propuesta independiente de estos autores, contribuyendo con la definición de los componentes de la Inteligencia Emocional. Sánchez Carlessi (1997) señala que las condiciones familiares, sociales y educativas tienen una marcada importancia con el desarrollo de la Inteligencia Emocional. Por todo lo mencionado entendemos que la formación integral del ser humaño es tarea de todos, pero formalmente de los profesores y de los padres de familia comprometidos con el cambio positivo y cualitativamente superior.

Karch y Witoreck (1995) refiere que la ayuda de los profesionales involucrados en la educación no debe estimular sólo patrones convencionales, sino facilitar una apertura hacia las actividades divergentes que fomenten un adecuado dinamismo personal. En este contexto y desde la perspectiva psicopedagógica y tutorial, consideramos importante enfocar los componentes interpersonales, intrapersonales, adaptabilidad, manejo del estrés y estado de ánimo en general.

La sociedad en la actualidad está atravesando por una grave crisis como consecuencia de la globalización que se aprecia no sólo en el plaño económico, sino también en el plaño educativo y, por ende, en los diversos niveles de enseñanza.

La personalidad se desarrolla a raíz del proceso de socialización, en la que el niño asimila las actitudes, valores y costumbres de la sociedad. Y son los padres, principalmente, los encargados de contribuir a esa labor, a través de su amor, cuidados, de la figura de identificación que para los niños (son agentes de socialización), es decir la vida familiar será la primera escuela de aprendizaje emocional.

La inestabilidad emocional es producto de la formación en el hogar, de la dinámica familiar inadecuada, de madres adolescentes, agresiones físicas entre padres y y de los padres a sus hijos, lo cual ha intensificado en problemas de conductas de los alumnos como: alcoholismo y drogadicción a temprana edad.

La regla importante en este sentido, tal como lo indican Elias Tobias y Friedlander (2000), es tratar a sus hijos como les gustaría que les tratasen los demás. Por tanto, en la escuela se debe inculcar a los alumnos a ser emocionalmente más inteligentes, dotándoles de estrategias y habilidades emocionales básicas que les protejan de los factores de riesgo o, al menos, que palien sus efectos negativos (Gea Rodríguez; 2001). Sin embargo, considerando que los docentes y padres de familia otorgan mayor importancia al área cognitiva, creemos conveniente trabajar en la presente investigación con las habilidades emocionales por cuanto se hace necesario aprender a otorgarle importancia a esta área emocional y hacer posible las relaciones que establecemos con los demás y así poder manejar mejor y solucionar los problemas que se generan. La Inteligencia Emocional es 
necesaria para enfrentar adecuadamente las dificultades que se presentan en el diario vivir, ello les otorgaría mayores niveles de satisfacción y de desarrollo personal, por cuanto el docente no deberá trabajar para el aquí y el ahora con los alumnos, sino para que sean en el futuro personas que se incorporen a la sociedad satisfactoriamente, tanto en su vida personal como laboral.

Un actuar bien es conocer bien el origen y la naturaleza de sus emociones y poder controlarlas de manera positiva, constituyéndose las más importantes emociones en el hogar y en la escuela y aprender a establecer relaciones adecuadas en sus pensamientos y que se expresen en sus comportamientos, es decir, desarrollando la Inteligencia Emocional. Goleman (1995) ha llamado a esta educación de las emociones "Alfabetización emocional" (también, escolarización emocional), y según él lo que se pretende con esto es enseñar a los alumnos a modular su emocionalidad desarrollando su Inteligencia Emocional.

Es por ello que se considera necesario y oportuno trabajar no sólo con los alumnos, sino en primera instancia con los padres de familia y docentes para motivarlos, es decir, enseñarles a manejar adecuadamente sus emociones, su autoestima y, sobre todo, su autoconfianza, dado que cuando el niño llega al colegio ya tiene una sólida imagen de sí mismo, que continúa desarrollándose en el marco social. El colegio ofrece la mejor oportunidad, después de la familia, para que el niño pruebe sus habilidades y se gane el respeto a los demás.

La inteligencia emocional es una de las habilidades para la vida, que debería enseñarse en el sistema educativo (Martín, 2001). En relación con los padres se incidió en que no sean muy permisivos ni autoritarios, deben darle a los niños la respectiva explicación con respecto a las respuestas que les den, sino harán de ellos niños egoístas o con bajo potencial de sociabilización; y lo que deseamos es que sean personas felices pues las emociones no controladas producen no sólo problemas emocionales, sino también problemas de salud. Es por ello que el programa le otorgó mucha importancia a la capacidad de empatía, ánimo positivo y tranquilidad.

\section{Programa para desarrollar la Inteligencia Emocional}

Como punto de partida se consideró a la escuela y su relación con la educación emocional, debido a los siguientes aspectos:

1. Porque repercute en las relaciones interpersonales, en el clima de la clase, en la disciplina, en el rendimiento académico, etc.

2. El conocimiento que tengan los docentes acerca de los componentes de la inteligencia emocional facilita la labor preventiva que puede ponerse en práctica cuando estamos dictando una clase.

3. La permanencia del niño en la escuela condiciona la formación del autoconcepto académico e interrelacional, en la que los individuos evalúan unos a otros: los profesores juzgan a los alumnos, estos se juzgan entre sí y a los profesores.

4. Cuando el niño llega al colegio ya tiene una primitiva y sólida imagen de sí mismo, que continúa desarrollando en el marco social. El colegio ofrece la mejor oportunidad, después de la familia, para probar sus habilidades y ganar el respeto de los demás. 
Se consideró conveniente en el programa utilizar el enfoque sistémico de los quince factores de inteligencia emocional y social de Baron (1997). Donde los componentes serían: Intrapersonal, Interpersonal, Adaptabilidad, Manejo del estrés, Estado de ánimo general, y los Subcomponentes, los quince factores reforzadores de los componentes (Figura N. ${ }^{\circ}$ 1).

Figura N. ${ }^{0}$ 1. Componentes de la inteligencia emocional.

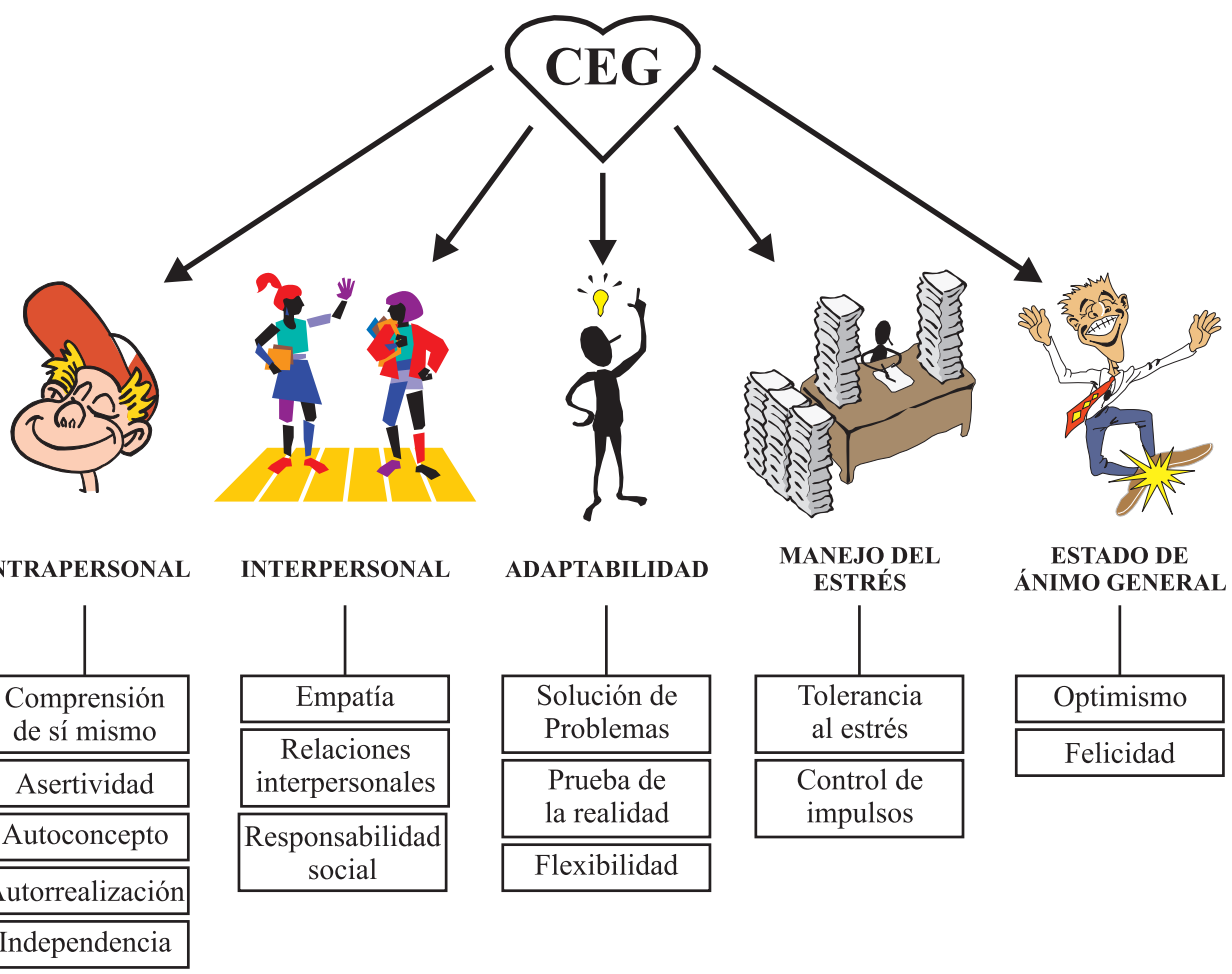

\section{Justificación del programa}

La finalidad de la educación emocional es desarrollar la personalidad integral en sus aspectos: cognitivo y emocional. Además la educación es un proceso caracterizado por la relación interpersonal y ésta involucra fenómenos emocionales.

\section{Objetivos del Programa}

- Reflexionar sobre los fines últimos de la educación y el rol del maestro, los cuales deben orientar el trabajo de las capacidades y valores.

- Establecer relaciones entre la educación y la inteligencia emocional de los alumnos. 
- Adquirir un mejor conocimiento de las propias emociones.

- Identificar las emociones de los demás.

- Desarrollar la habilidad de controlar las propias emociones.

- Prevenir los efectos perjudiciales de las emociones negativas.

- Desarrollar la habilidad para generar emociones positivas.

- Adoptar una actitud positiva ante la vida.

- Compartir y definir estrategias que nos permitan trabajar en el aula una educación que trascienda.

Los requisitos para participar en el programa de intervención fueron:

- $\quad$ El profesorado que participa en la educación emocional debe sentirse cómodo hablando de las emociones.

- Una vez iniciado el programa se trata de reflexionar acerca del mismo. ¿Cómo está funcionando?, ¿cómo podría mejorarse?, ¿cómo resolver las dificultades? Hay que realizar una evaluación continua durante el proceso de aplicación.

Los aspectos considerados en el desarrollo de las emociones fueron los siguientes:

- Los alumnos deberían tener edades comprendidas entre los 10 y 11 años.

- Su rendimiento académico debería ser satisfactorio, en la medida que produce alta autoestima, en tanto que bajo rendimiento académico produce lo contrario, actitudes negativas, tristeza, pesimismo, incluso depresión.

- El alumno debería tener la habilidad de posponer gratificaciones inmediatas a favor de otras gratificaciones de orden superior, es un factor de inteligencia emocional que conviene potenciar en estas edades.

- En el último grado de Primaria se puede introducir la figura del "mediador" en los conflictos. La mediación se propone, en primer lugar, prevenir la violencia. Esto supone la formación y entrenamiento en mediación y negociación.

- El alumno debería tener la capacidad de reconocer cómo se siente uno, cómo controlar los impulsos, acción tan importante para evitar la violencia, y cómo manejar la furia.

\section{Contenidos de la educación emocional trabajados}

En el desarrollo del programa se trabajaron los siguientes aspectos:

- Evaluación de las emociones.

- Identificación y reconocimiento de las emociones negativas.

- Identificación y reconocimiento de las emociones positivas.

- Analizando ¿cómo se sienten los demás? 
- Aprendiendo las habilidades emocionales.

- Aprendiendo las habilidades de comunicación.

\section{MÉTODO}

En el diseño de la investigación, se utilizó un diseño cuasiexperimental con grupo de comparación, pues se trata de estudiar el efecto del programa sobre el desarrollo de la inteligencia emocional en los alumnos de sexto grado de primaria. (Hernández, Fernández y Baptista, 2006).

Universo y muestra de Investigación. El universo estuvo conformado por alumnos de sexto grado de primaria, matriculados en el 2005 en colegios de gestión estatal y no estatal de la ciudad de Lima. Se trabajó con una muestra de 80 estudiantes: 40 de colegios estatales y 40 de colegios no estatales; en cada caso 20 conformaron el grupo de control. Para la obtención de la muestra, se aplicó un procedimiento de muestreo intencional por cuotas.

\section{Variables de estudio}

\section{Variables Independientes}

- Programa experimental

- Tipo de gestión del colegio de procedencia

\section{Variables Dependientes}

- Desarrollo de la Inteligencia Emocional

Técnica e instrumentos de recolección de datos. En la investigación se aplicó el inventario de inteligencia emocional de Baron ICE:NA, instrumento que fue adaptado a nuestro contexto por Ugarriza (2003), el cual ha demostrado que presenta validez y confiabilidad.

Procedimiento de recolección de datos, se realizó los siguientes pasos: en primer lugar se aplicó el inventario como un pretest antes de aplicar el programa. En segundo lugar se aplicó el programa experimental que consistió en:

- Implementación de un programa dirigido a los alumnos en el cual se trabajó las habilidades y competencias que constituyen la Inteligencia Emocional.

- Se organizó un ciclo de conferencias para los docentes sobre la inteligencia emocional y su importancia en el trabajo con los alumnos.

- Teniendo en cuenta la importancia de la participación activa de los padres de familia se organizó un ciclo de conferencias para los docentes sobre inteligencia Emocional y su importancia en el trabajo con los alumnos.

Al finalizar el programa se aplicó un postest. 
Técnicas de procesamiento y análisis de datos, el análisis estadístico fue realizado tomando en cuenta las recomendaciones propuestas por Siegel y Castellan (1995), el cual abarcó los siguientes tipos de análisis:

A. Análisis descriptivo, para describir la forma de distribución de las variables cuantitativas a través de las pruebas de bondad de ajustes a la curva normal de Kolmogorov Smirnov para los puntajes a las escalas aplicadas.

B. Análisis Inferencial para la contrastación de hipótesis, se ejecutó las pruebas Z de diferencias de medias para grupos independientes para comparar entre los grupos experimental y control, el tipo de gestión de colegio y el sexo de los alumnos.

\section{RESULTADOS}

\section{Etapa I. Análisis descriptivos}

Los resultados de los análisis de la bondad de ajuste a la curva normal de las variables estudiadas, fueron realizados través de la prueba de Kolmogorov-Smirnov (ver tabla N. ${ }^{\circ}$ 1), los hallazgos permiten apreciar que en todos los casos los valores que se alcanzaron en el estadísticos Z de Kolmogorov-Smirnov no son significativos, por lo que se puede concluir que las escalas del inventario de Inteligencia Emocional de Baron, presentan una adecuada aproximación a la curva normal. Es por ello que se utilizan contrastes estadísticos paramétricos en el análisis de los datos de la investigación (Siegel y Castellan, 1995, Elorza, 2007).

Tabla N. ${ }^{0} 1$. Prueba de bondad de ajuste a la curva normal de Kolmogorov-Smirnov de las variables estudiadas.

\begin{tabular}{lccc}
\hline \multicolumn{1}{c}{ Variable } & Media & Desviación típica & $\begin{array}{c}\text { Z de } \\
\text { Kolmogorov-Smirnov }\end{array}$ \\
\hline Escala intrapersonal & 107.43 & 13.89 & 1.00 \\
Escala interpersonal & 98.30 & 15.86 & 1.05 \\
Escala de manejo del estrés & 109.38 & 10.93 & 0.95 \\
Escala de adaptabilidad & 100.67 & 15.82 & 1.22 \\
Escala de ánimo en general & 90.83 & 34.16 & 1.08 \\
Puntaje total & 104.30 & 17.95 & 1.02 \\
\hline
\end{tabular}

$\mathrm{N}=80$

\section{Etapa II. Análisis inferencial del contraste de hipótesis}

Inicialmente se planteó la primera hipótesis teórica, la cual propone que los alumnos que participan en el programa mejorarán su Inteligencia Emocional. Para ello se comparó los puntajes contenidos en el Inventario de Coeficiente Emocional de Baron entre el grupo experimental y el grupo de control, a través de la prueba $\mathrm{Z}$ para dos grupos independientes. 
Los resultados indicaron que existen diferencias estadísticas significativas en la Escala Intrapersonal $(Z=2.54 \mathrm{p}<.05)$, La Escala Interpersonal $(Z=282 \mathrm{p}<.01)$, Escala de Manejo del Estrés $(Z=2.39 \mathrm{p}<05)$, la Escala de Adaptabilidad $(3.57 \mathrm{p}<.01)$, la Escala de Ánimo en General $(Z=3.19 \mathrm{p}<.05)$ y en el Puntaje Total (Puntaje Total $(Z=2.61 \mathrm{p}<.05)$. Notándose que en todos los casos el grupo de experimental alcanzó puntajes más elevados que el grupo de control.

En conclusión los hallazgos indican que la Hipótesis 1 es válida, pues los alumnos que perticiparon en el programa experimental alcanzaron un mejor puntaje en las escalas del Inventario de Inteligencia Emocional de Baron.

Tabla N. ${ }^{\circ}$ 2. Análisis comparativo por grupo de estudio.

\begin{tabular}{|c|c|c|c|c|c|}
\hline \multirow{2}{*}{ Variable } & \multirow{2}{*}{$\begin{array}{c}\text { Experimental } \\
M\end{array}$} & \multirow{2}{*}{$\begin{array}{l}\mathrm{N}=\mathbf{4 0} \\
\text { D.E. }\end{array}$} & \multicolumn{2}{|c|}{ Control $N=40$} & \multirow{2}{*}{$\mathbf{Z}$} \\
\hline & & & $\mathbf{M}$ & D.E. & \\
\hline Escala intrapersonal & 111.25 & 9.68 & 103.62 & 16.34 & $2.54 *$ \\
\hline Escala interpersonal & 103.09 & 9.75 & 93.52 & 19.17 & $2.82 * *$ \\
\hline Escala de manejo del estrés & 112.22 & 6.56 & 106.54 & 13.50 & $2.39 *$ \\
\hline Escala de adaptabilidad & 106.56 & 11.85 & 94.77 & 17.19 & $3.57 * *$ \\
\hline Escala de ánimo en general & 102.35 & 21.17 & 79.30 & 40.53 & $3.19 *$ \\
\hline Puntaje total & 109.35 & 11.74 & 99.25 & 21.51 & $2.61 *$ \\
\hline
\end{tabular}

$* \quad \mathrm{P}<.05 * * \mathrm{p}<.01$

Con relación a la segunda hipótesis teórica propuesta, la cual indica que existen diferencias entre los alumnos según el tipo de colegio de procedencia. En la tabla N. ${ }^{\circ} 3$ se encuentra que los hallazgos indican que no existen diferencias estadísticas significativas en escala alguna. Por ello se concluye que la segunda hipótesis teórica no es válida y los alumnos de colegios estatales y particulares presentan niveles similares en las escalas de la inteligencia emocional.

Tabla N. ${ }^{0}$ 3. Análisis comparativo por tipo de gestión del colegio.

\begin{tabular}{|c|c|c|c|c|c|}
\hline \multirow{2}{*}{ Variable } & \multicolumn{2}{|c|}{ Estatal $N=40$} & \multicolumn{2}{|c|}{ Particular $\mathrm{N}=\mathbf{4 0}$} & \multirow{2}{*}{$\mathbf{Z}$} \\
\hline & M & D.E. & M & D.E. & \\
\hline Escala intrapersonal & 102.04 & 15.54 & 103.12 & 17.08 & -0.30 \\
\hline Escala interpersonal & 90.26 & 20.36 & 93.94 & 18.33 & -0.85 \\
\hline Escala de manejo del estrés & 102.62 & 12.26 & 104.35 & 15.62 & -0.55 \\
\hline Escala de adaptabilidad & 96.42 & 14.82 & 94.75 & 17.03 & 0.47 \\
\hline Escala de ánimo en general & 11.57 & 31.70 & 19.66 & 40.35 & -1.00 \\
\hline Puntaje total & 96.95 & 18.59 & 98.86 & 18.90 & -0.46 \\
\hline
\end{tabular}


Respecto a la tercera hipótesis teórica propuesta, en la que se propone que existen diferencias en el manejo de la inteligencia emocional, según el sexo de los alumnos, los resultados presentados en la tabla N. ${ }^{\circ} 4$ indican que no existen diferencias entre los alumnos según el tipo de colegio de procedencia. En la tabla N. ${ }^{\circ} 3$ se encuentra que los hallazgos indican que no existen diferencias estadísticas significativas en escala alguna, se concluye que la hipótesis teórica no es válida y los alumnos de colegios estatales y particulares presentan niveles similares en las escalas de la inteligencia emocional.

Tabla N. ${ }^{\circ}$ 4. Análisis comparativo por sexo.

\begin{tabular}{|c|c|c|c|c|c|}
\hline \multirow{2}{*}{ Variable } & \multicolumn{2}{|c|}{ Varón $\mathrm{N}=\mathbf{4 0}$} & \multicolumn{2}{|c|}{ Mujer $\mathbf{N}=\mathbf{4 0}$} & \multirow{2}{*}{$\mathbf{Z}$} \\
\hline & $\mathbf{M}$ & D.E. & $\mathbf{M}$ & D.E. & \\
\hline Escala intrapersonal & 105.81 & 14.36 & 108.70 & 13.53 & -0.92 \\
\hline Escala interpersonal & 98.93 & 14.62 & 97.82 & 16.90 & 0.31 \\
\hline Escala de manejo del estrés & 110.34 & 13.71 & 108.63 & 8.24 & 0.69 \\
\hline Escala de adaptabilidad & 100.76 & 17.00 & 100.59 & 15.04 & 0.05 \\
\hline Escala de ánimo en general & 94.11 & 31.96 & 88.27 & 35.92 & 0.76 \\
\hline Puntaje Total I. E. & 104.64 & 19.99 & 104.04 & 16.42 & 0.15 \\
\hline
\end{tabular}

\section{CONCLUSIONES}

1. Las escalas del inventario de Inteligencia Emocional de Baron. Presenta una adecuada aproximación a la curva normal.

2. La hipótesis 1 es válida, los alumnos que participaron en el programa experimental alcanzaron un mejor puntaje en las escalas del Inventario Emocional de Baron.

3. La hipótesis 2 no es válida, no existen diferencias al comparar las escalas del Inventario Emocional de Baron según los tipos de gestión del colegio de procedencia.

4. La hipótesis 3 no es válida, no existen diferencias al comparar las escalas del Inventario Emocional de Baron por sexo.

5. El programa fue eficiente para incrementar la Inteligencia Emocional en los alumnos que participaron en el grupo experimental.

6. El personal docente con el cual se trabajó aplica de manera positiva y adecuada la inteligencia emocional, utilizando el material proporcionado y mostrando interés para continuar con el proyecto

\section{REFERENCIAS BIBLIOGRÁFICAS}

1. Baron, R. (1997). Baron Inventario Emocional. México: Selecta.

2. De Bono, Edward (2000). Pensar bien, utilice al máximo su potencial intelectual y creativo de la mente. México: Selecta 
3. Elías, M. J., Tobbias, S. E., y Friedlander, B. S. (2000). Educar con Inteligencia Emocional. Barcelona: Plaza Janés.

4. Elorza, H. (2007). Estadística para las ciencias sociales, del comportamiento y de la salud. México: CENGAGE Learning.

5. Gardner, H. (1995). La inteligencia reformulada. Las inteligencias múltiples en el siglo XXI. Barcelona: Paidós.

6. Goleman, D. (1995). Inteligencia emocional. Barcelona: Cairos.

7. Hernández, R., Fernández, C. y Baptista, P. (2006). Metodología de la Investigación. México: Mc Graw-Hill.

8. Martin, A. y Marchesi, E. (2001). Calidad de la enseñanza en tiempos de cambio. Madrid: Alianza.

9. Salovey, P. y Mayer, J. D. (1990). Emotional intelligence. Imagination, Cognition, and Personality, 9 (3), 185-211.

10. Sánchez Carlessi, H. (1997). Revista de Psicología. Universidad Ricardo Palma.

11. Siegel, S. y Castellan, N. (1995). Estadística no paramétrica: Aplicada a las ciencias de la conducta. México: Editorial Trillas.

12. Ugarriza CH., N. y Pajarez D. A., L. (2005). Adaptación del Inventario Emocional BARON ICE:NA completo. Lima (S/E). 